\title{
The Role of Nutrition Counseling in Controlling the Non-Communicable Diseases among Nepali Women
}

\author{
${ }^{1}$ Kabita Maharjan \\ ${ }^{2}$ Deepak Chaudhary \\ ${ }^{1}$ Nepal Cancer Hospital \& Research Centre, Lalitpur, Nepal \\ ${ }^{2}$ International Environmental Solution \& Management, Kathmandu, Nepal
}

Article History: Submitted 04 February 2021; Reviewed 21 April 2021; Accepted 30 April 2021

Corresponding Author: Kabita Maharjan, Email: kdkabs@gmail.com

DOI: https://doi.org/10.3126/paj.v4i0.37053

Copyright 2021 ( ) Author/s and Centre for Research and Innovation.

This work is licensed under a Creative Commons Attribution 4.0 International (CC BY 4.0) License.

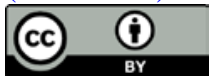

\section{ABSTRACT}

The general perception of nutrition is that it is just the process of taking food. However, it represents food science that ultimately relates to human health. Unhealthy and imbalanced food and the modern lifestyle are contributing to non-communicable diseases (NCDs) in recent days. Hypertension, diabetes and cancer are some major NCDs across the world that have created challenges for human life. Annually, millions of people die due to the food related problems. The major objective of the study is to analyze the health status of non-communicable diseases among Nepali women. This study emphasizes the counseling and modification of diet that are crucial in controlling non-communicable illness. This study is based on the secondary data and review of literature. All these data were reviewed through journals, research articles, books, and the association's dietary guidelines. The reports by WHO, Nepal Demographic Health Survey, American Heart Association, American Lung Association and National Institute of Diabetes are also reviewed. The experience of working in this field for a long time has also been utilized in analyzing the data. A number of NCDs is being increased globally and Nepal is no exception. The WHO report of 2018 reveals that NCDs are killing 41 million people yearly, equivalent to $71 \%$ of all death globally. Similarly, 15 million people die from it between 30 and 69 age groups, and 85\% of these 'premature' deaths in low and middle-income countries. According to NDHS (2016), the prevalence of hypertension in women in Nepal is 17\% of those aged 15 and above. Alter food habits, overweight and obesity as well as food practices during pregnancy, after delivery and daily intake of convenience fast food in Tiffin and snacks are some reasons to increase the NCDs. Nutrition counselling and awareness, dietary policy guidelines and an active role of diet specialist (dietician/nutritionist) will be the effective mitigation in controlling the NCDs number.

KEYWORDS: Counseling, lifestyle, food, nutrition, non-communicable diseases 


\section{INTRODUCTION}

Nutrition is fundamental to human health and life. Food is one of the components of human holistic development. Nature has provided a variety of foods in terms of nutrition. The combination of food in the right amount is known to be a balanced diet. So, it is essential in order to meet the daily requirement as per gender, physical activities, ecological region and physiological condition.

The concept of balanced nutrition has existed since the period of Aristotle and Galen. Scribonius Largus emphasized the importance of diet along with medical treatment (Galen, 2000). It is a famous saying of Hippocrates- "Let food be the medicine and medicine be thy food." Over time, modern nutrition science has a versatile role in nutritional-related non-communicable diseases (NCDs). World Health Organization $(2018$, a) defines it as chronic diseases, which tend to be of long duration and are the result of a combination of genetic, physiological, environmental and behavioral factors. Non-communicable diseases (it is known as NCDs) are the diseases that are not transmitted from one person to another person. The major NCDs are cardiovascular disease, cancers, chronic respiratory diseases and diabetes. The WHO report reveals that NCDs excessively affected peoples belonging to low and middle-income countries where more than three-quarters of global NCD deaths occur. Unfortunately, 15 million people belong to low and middle-income developing countries aged 30 and 69 of premature death due to NCDs. In the global context, the death rate of NCDs per year is 41 million corresponding to $71 \%$ of global deaths. Among many NCDs, one that relates to the heart known as cardiovascular disease, and people die about 17.9 million. The WHO report shows that the death from NCDs in Nepal is 65\% out of 1,21,000 and the risk of premature death is 22\%. Similarly, the Nepal Demographic Health Survey (NDHS, 2016) report shows the prevalence of hypertension aged 15 and above in Nepali women is $17 \%$.

Cardiovascular diseases account for most NCDs deaths, or 17.9 million people annually, followed by cancers (9.0 million), respiratory diseases (3.9million), and diabetes (1.6 million). Likewise, Nepal cannot be exceptional from it. According to NDHS (2016), the hypertensive women percentage aged 15 to 69 is $15 \%$. Similarly, the rates of hypertension among tobacco user women are $16 \%$ whereas those who do not use tobacco are $10 \%$. Moreover, the percentage of hypertensive is $38 \%$ which is twice higher in obese women age 15 or above. Comparatively in Province 4, hypertension in women is $24 \%$ aged above 15 . It implies the growing trend of NCDs and needs to be further inquired for preventive measures so that they could contribute to a quality life. Recently, the Covid-19 highlights the nutrition diet for immunity power (Chaudhary et al., 2021). The role of nutrition counseling and diet is critical in the wake of reducing such diseases.

\section{METHODS}

This study aims to analyze the importance of nutrition in minimizing the impact of NCDs. This is a review article based on the secondary data. Nutrition includes food patterns and food habits. All these data are reviewed through journals, research articles, books and dietary guidelines. For it, the reports and guidelines prepared by WHO, Nepal Demographic Health Survey, American Heart Association, American Lung Association, National Institute of Diabetes Digestive and Kidney Disease, Ministry of Health and Population Nepal (MoHP) are intensively reviewed. This study primarily focuses on Nepali women because of the increasing disease trend in them. 


\section{SIGNIFICANCE OF STUDY}

The study emphasizes nutritious foods based on the Nepali context scientifically that could be a way out for improving food habits and is concerned with women's health particularly. According to the WHO, the reports of the past three decades showed that NCDs are one of the death causes among women globally and this number has been defined as two in every three deaths each year, especially in low and middle-income countries like Nepal. In the context of Nepal, poverty in rural Nepal represents 27\% which is still higher than urban areas (15\%) (Chaudhary, 2018). Similarly, it is a misperception that NCDs especially cardiovascular disease is the primary disease of men. In the same way, another misconception is that NCDs occur in women of highincome countries. So, it is required for specialized personnel as Dieticians in the management of clinical nutrition. Dieticians are the core person in the wake of food science who cares about food and nutrition, its quantity to be taken based on the principle of disease, and patient health condition. There is a great role of nutrition to human beings either to stay healthy or to control disease conditions. Nutritional deficiencies have been caused many health problems in Nepal (Maharjan \& Chaudhary, 2012). Therefore, this paper will support patients suffering from NCDs following preventive measures on a diet, policymakers and dietician personnel.

\section{FINDINGS AND DISCUSSION}

Globalization and modernization have greatly contributed to changing patterns globally, especially in developing countries. A female has to bear a child, so they belong to the vulnerable group because the woman/mother's nutritional status impacts the fetus's health. Women can be compared with nature; they can generate a new generation. Thus, good nutrition is essential for women and children for the sustainability of human life. Development and education have changed women to dissolve the outer society. At an early age, all the women take care of family and household activities. To date, women come foremost taking care of family and home. Women are facing challenges in balancing family and professional responsibilities i.e. both in-home and workplace. In a short period, the female has to manage household activities. So, females are compelled to add convenience food despite knowing realities. However, children have fond of having convenience food.

Convenience food advertisements and attractive wrappers magnetized many people. It does not need preparation; in the absence of parents, children can have those foods easily whether they feel hungry or not. The nutritive value coded on the wrapper is about food information. Most convenience foods are calorie-dense and these foods tend to put on unexpected and unnecessary weight if taken excessively. Today most of the children are overweight and obese; this might be one of the reasons behind it. However, these foods became a part of life. So, while consuming or buying, one should be conscious of nutritive value and ingredients content.

\section{NCDs Related to Human Lifestyle and National Nutrition and Health Policy Smoking and alcohol}

Smoking and alcohol detriment both male and female's health. Despite it, people used to take at their own risk. Smoking is a major factor for lung cancer. In the context of Nepal, the traditional practice of cooking has also been contributed to smoke i.e. environmental pollution. The indoor household cooking smoke is found $29.8 \%$ in an urban area and 33.8\% in rural; overall, it leads 31.3\% (NHDS, 2016). It indicates that in the coming days the number of lung cancer might be increased. Not only direct smoke but indirect smoke too also hazardous for health. Second-hand smoke enclosed more 
than 7000 chemicals; among them, 100 is toxic and nearly 70 can cause cancer. The nonsmoker exposed at home or outside may increase the risk of developing lung cancer by $20-30 \%$. The pregnant women are exposed to secondary smoke; then it may cause Sudden Infant Death Syndrome (SIDS) in the child. The children may suffer from wheezing, coughing and asthma attack and in the case of an infant, there may be unexplained and unexpected death in the first year of life. Besides these parents who smoke, children may suffer from an ear infection, accumulation of fluids and sometimes may need operation to put ear tubes for drainage (CDC, 2018).

The Nepal government has the Tobacco Product Act (2010) to make people more aware of the negative effects of smoking; certain restrictions are considered as smoke-free place, tobacco advertising, promotion and sponsorship, tobacco packaging and labeling, a roadmap to tobacco control legislation, which are the steps against smoking. Despite a larger advertisement against smoking, the consumption of smoking is not being declined. The smoking trend is higher among lower socio-economic groups rather than the higher income class. According to NDHS (2016), the prevalence of hypertension in Nepali women is $17 \%$ of those aged 15 and above. Regionally, Gandaki Province represents the highest number of hypertension cases as it stands $24 \%$ of aged between 15 and 69 whereas Sudur Pachhim Province represents the lowest one as it stands for $15 \%$ only. Similarly, $16 \%$ of women who use tobacco have hypertension and $10 \%$ of women have hypertension without smoking. This result shows that smoking is one of the causes of hypertension.

Likewise, alcohol provides seven kilocalories per milliliter. It is calorie-dense comparing to mandatory nutrients as carbohydrate and protein, which provides four kilocalories per gram. There is a variation of alcohol-containing percentage in alcoholic drinks like wine, whisky, beer, rum, scotch, etc. According to the CDC (2019), it has been warned that an excessive intake of alcohol can cause cancer. When alcohol is taken, the body produces a chemical substance called acetaldehyde, which unknowingly starts to damage cells. Men who take more than five serving and female more than four serving may cause serious health problems as injuries, violence, liver disease and even cancer (CDC, 2017). Cao (2015) explains that alcohol helps in changing estrogen pathways, thus increasing the risk of developing breast cancer in females. Even a moderate amount of alcohol intake showed the risk of developing breast cancer in females (Liu, 2015).

According to the Heart Foundation, hypertension is one of the most common health problems in the world. $120 / 80 \mathrm{mmHg}$ is considered normal blood pressure and more than $140 / 90 \mathrm{mmHg}$ as hypertensive. There is a not significant reason for hypertension but significant root causes as not getting enough physical activities, drinking more than two servings of alcoholic drinks, stress, and old age, smoking, and a family history of high blood pressure (Heart Foundation). This shows that there is an indirect association between alcohol and hypertension. As NDHS (2016), the hypertension rate is found twice among obese women who scored $38 \%$ of 15 years and above age group. Nepal Health Sector has developed a strategy to reduce hypertension from $26 \%$ to $22 \%$ by 2020 . 


\section{Hypertension among Different Age Women}

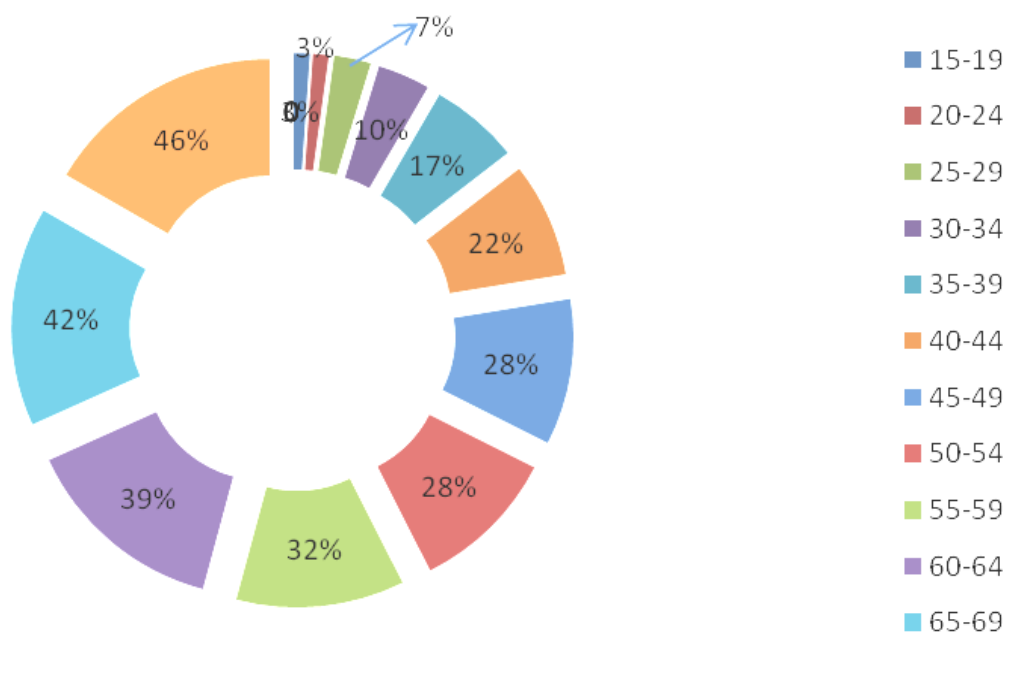

Fig. 1. Hypertension among Different Age Women, NDHS 2016

\section{Overweight and Obesity}

WHO has mentioned that overweight and obese are an alarming condition of encountering NCDs. According to NDHS (2016), comparing to the reports of 2006, 2011 and 2016, it reveals that overweight and obesity due to over nutrition has been contributed to a higher number of as it represents $9 \%, 13 \%$ and $22 \%$ respectively. This percentage shows an increasing trend in overweight and obesity. Similarly, the nutritional status of women shows $5 \%$ obese, $17 \%$ overweight, $61 \%$ normal weight and $17 \%$ are thin respectively. Among many factors of being overweight, food types, food frequency and dietary patterns are significantly correlated with BMI (Maharjan, 2019).

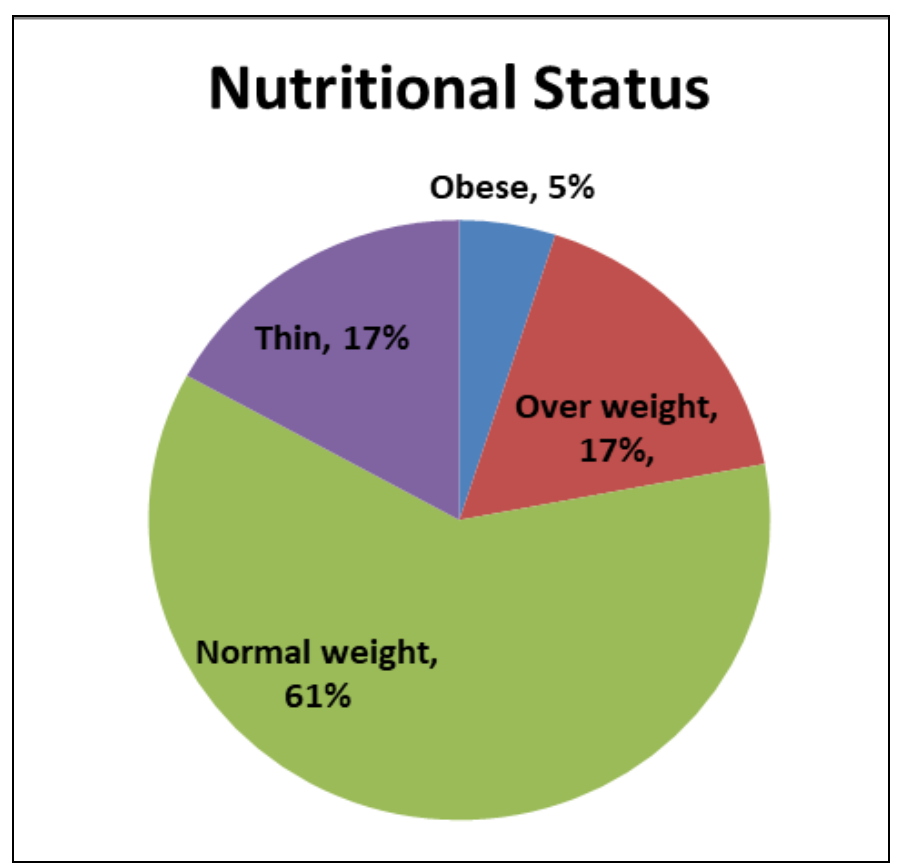

Fig. 2. Nutritional Status of Nepalese Women, NDHS 2016

Prithvi Academic Journal, Volume 4, May 2021 [pp. 121-132] 125 | P a g e 
The NDHS (2016) report reveals that hypertension is being increased with a higher Body Mass Index (BMI). According to American Heart Association (AHS, 2020), it has been categorized BMI as thin $(<18.5 \mathrm{~kg} / \mathrm{m} 2)$, Normal $(18.5-24.9 \mathrm{~kg} / \mathrm{m} 2)$, overweight $(>25.0 \mathrm{~kg} / \mathrm{m} 2)$ and obese $(>30 \mathrm{~kg} / \mathrm{m} 2)$. The report reflects that the socioeconomic status has large impacts on increasing NCDs. The report classified socioeconomic in five categories as lowest, second, middle, fourth and highest. The lowest income group has $15 \%$ hypertensive cases and the highest group showed $24 \%$. This data showed hypertension is higher in the wealthiest family rather than the poorest family. BMI is one of the indicators of nutritional status linked with hypertension. Figure 3 shows BMI higher than the hypertension percentage.

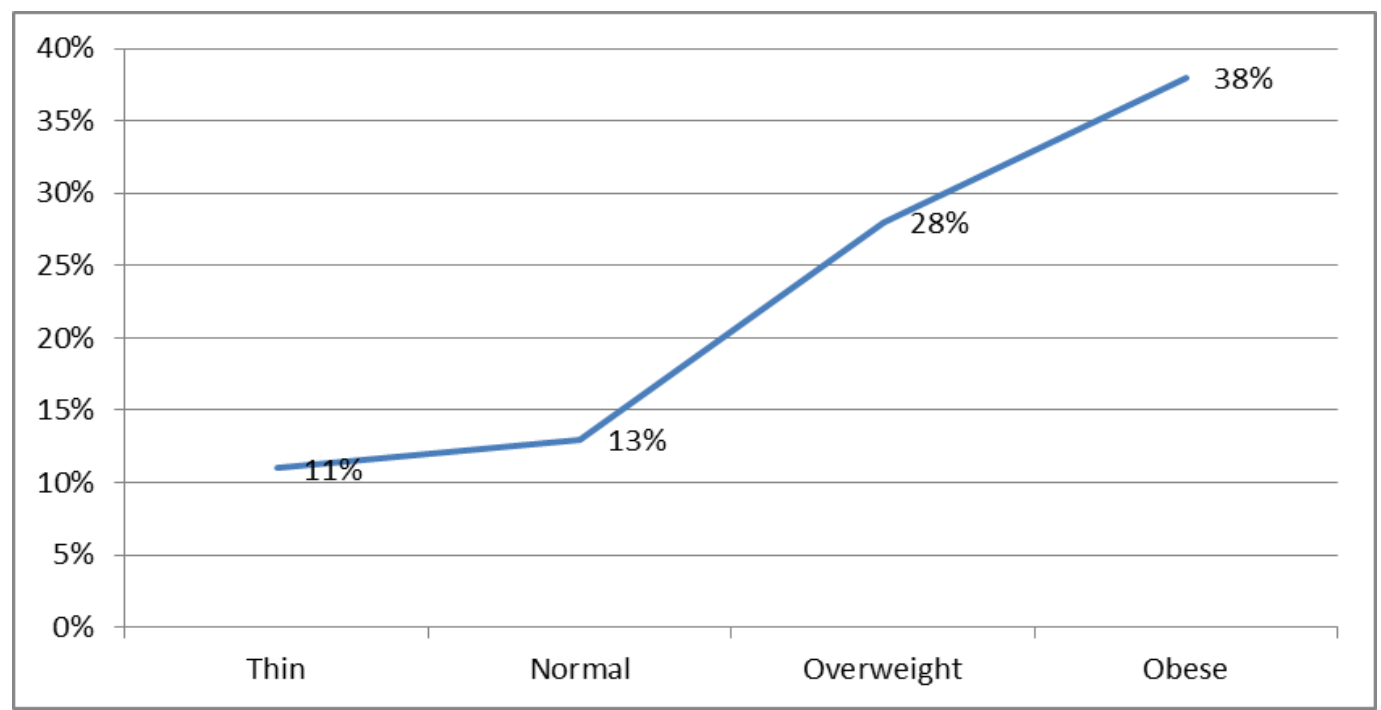

Fig. 3. Hypertension Scenario based on Body Mass Index (BMI) in Nepal, NDHS 2016

Overweight and obesity are increasing in Nepali women as well. According to WHO (2018), the worldwide prevalence of obesity is nearly tripled compared to the data between 1975 AD to 2016 AD. The WHO reports of 2016 show that people aged over 18 years were more than (1.9 billion) overweight among over 650 million adults who were obese. The overweight population in the world is $13 \%$. Among this, $15 \%$ of women are obese. The overweight population among adults aged 18 years is $39 \%$ and the women's overweight population is $40 \%$.

So, the importance of nutrition awareness may be pivotal in minimizing NCDs. The study published by a European Heart Journal mentioned that NCDs are related to human lifestyles. Global change and preventive measures play an important role in controlling NCDs. The various health associations have developed dietary guidelines to prevent NCDs' diseases. The strong policy should be amended for better health country people (European Heart Journal). The study of Naya (2017) followed descriptive and experimental research methods in the quantitative design. It was done in Arabian countries between 2006 and 2015. The sample population of the study was 824. The study focused on dietary patterns, food frequency, and dietary assessment to study nutritional assessment. Different studies showed that the ratio of NCDs is higher in middle and low income in developing countries; however, there should conduct nutrition research to find the exact situation of increasing and comparing the ratio. 


\section{Alteration of Food Habit}

Convenience food like biscuits, noodles and snacks becomes an integral food in our day to day life. Most people start the day with tea and biscuits. In these convenience food wrappers, it is mentioned of nutritive value. So, it will be excellent if the consumers go through nutritive value whether the food is good or not for one's health. Taking food is not just of taste and quench hunger, it should be nutritious for health. In the context of Nepal, it is a very complex thing about food quality; time and again the news is flashed against the quality of food while monitored/surveillance.

The Nepal government has developed a food pyramid of five food groups (Figure 4) to meet the daily nutritional requirements that can help in preventing nutritional deficiency diseases. During the survey, it was found that the additional food during pregnancy is meat, egg and dark green leafy vegetables to meet the daily requirement of mother and fetus. However, these concepts are not well followed because of low income, inadequate awareness and cultural values. It is well said that nutrition deficiency not only affects the mother's health, but the child's health, too. The Nepal government has developed five food groups pyramid including cereals and starchy vegetables like potato, yam (group 1), pulses and legumes (group 2), vegetables and fruits (group 3), meat, egg, milk and milk products (group 4) and oils, sugar and nuts (group 5) in order to meet all the macro and micronutrients. It can easily guide to manage food groups even unable to read and write. The pictures below as shown in figure 4 are the food pyramid that is well defined in every food group based on the nutrients contained.

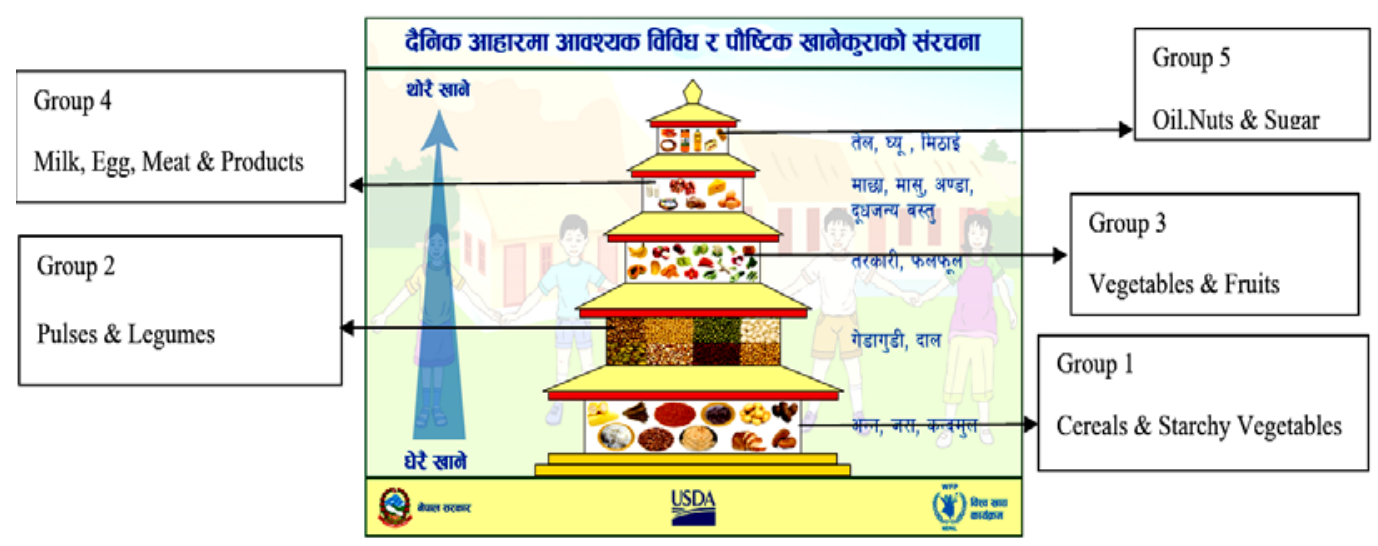

Source: Food Temple Poster-Nepali

Fig. 4. Nepali Food Pyramid for a Balanced Diet Model

Table 1

Additional Food for Women during Pregnancy and Breastfeeding Period

\begin{tabular}{llccc}
\hline S.No. & Food Particulars & \multicolumn{3}{c}{ Geographical Regions } \\
& & Hill & Mountain & Terai \\
\hline 1 & Meat consumption & $39 \%$ & $19 \%$ & NA \\
2 & Egg consumption & $19 \%$ & $10 \%$ & $10 \%$ \\
3 & Dark green leafy vegetables & $58 \%$ & $60 \%$ & $45 \%$ \\
4 & Consumption of 5 or more food & $58 \%$ & $53 \%$ & $28 \%$ \\
& group & & & \\
\hline
\end{tabular}

Source: NDHS, 2016.

Prithvi Academic Journal, Volume 4, May 2021 [pp. 121-132] 
The Nepal government has launched the program on Maternal, Infant, and Young Child Nutrition (MIYCN). This program has mobilized the community female health volunteer, mother's group and the civil society that encouraged to intake at least one meal extra meal per day and two meals during breastfeeding. Table 1 shows the entire food pattern that is lower in Terai comparing to Hill and Mountain area if comparing the geographical basis of Nepal. It might cause the Anemic; the prevalence of Anemia in Terai is $60 \%$, followed by Mountain $57 \%$ and Hill $40 \%$. The study carried out by Stuetz et al. (2019) states that inadequate consumption of vegetables and food habits that are dense in micronutrients can cause Anemia and overweight (Stuetz, 2019). Similarly, meat and egg consumption are found less, which are considered more nutritious food for fetus growth and development. A woman should change her diet pattern after delivery of six months till the child reaches two years. However, women's food habit remains the same intake meat amount high. In Nepali culture, people cook meat spicy and oily, the excessive oil increases calorie and excessive spice may imbalance electrolytes, which impacts health. So, diet modification should be done before, during pregnancy, and post-delivery. The meat consumption rate was higher in hilly region women and no intake among Terai women.

\section{Importance of Nutrition Awareness and Education}

In the context of Nepal, there are various impacting factors such as awareness regarding nutritional education that need to be improved. Nutrition awareness assists in the basic concept of food. According to NDHS (2016), the literacy rate is significantly poor among Nepali females as compared to males. The study age was 15 to 49 years. $50 \%$ of women had completed secondary or higher education. 1/3 of women had no education. Only $69 \%$ of women are literate. In urban women, $57 \%$ have completed at least secondary level education. This study was done at the age of 15 to 49 years of women.

Education enlightens all; the nutrition education method will be different in educated and uneducated people. It can be easily carried out in the day-to-day life. There should be a planned program for literate and illiterate women differently even the program theme is the same. However, nutrition education is equally important whether able to read or not. The proportion of women consuming five or more food groups is higher among women having a higher level of education (73\%) compared with women having no education (34\%). Similarly, women with good income have a higher consuming quantile (76\%) whereas lower quantile (38\%) among low-income women (NDHS, 2016). As CBS's (2011) relationship between education and income is imperative and both these support in affording nutritious food that is good for health. It is well-defined that metabolic changes are the threshold point for increasing NCDs risk on raised blood pressure, overweight/obesity, hyperglycemia, and hyperlipidemia (WHO, 2018b).

\section{Existing dietary policies and guidelines}

According to American Dietary Guidelines 2015-2020, in a regular diet, one should add a variety of high fiber whole grains, fruits and vegetables instead of highcalorie food with less salt 2300 milligram (mg), and in case of hypertension $1500 \mathrm{mg}$ is recommended and additional simple sugar. Skinless poultry is a good source of protein and fish as trout contains omega-3 fatty acid, which is available in the Nepali market and should consume without adding saturated fat and trans fat. If you want to have red meat, then you need to choose the leanest cuts and should be prepared in a healthy way. Fatfree or low fat $1 \%$ dairy products skim milk and products are preferable. Foods 
containing hydrogenated vegetable oil should be reduced for cardio health. It will be better to avoid any kind of hard drinks. However, two exchange for male and one exchange for a female is recommended.

In India, the National Institute of Nutrition (NIN) has formulated dietary guidelines to make everyone healthy. It has been described what should be eaten and how much to be taken in a day in terms of gender, physical and physiological condition. The Ministry of Health and Population Nepal (MoHP, 2018) has developed the video in order to an aware population in discouraging alcohol, smoking and tobacco chewing, regular exercise, healthy diet, regular health check-up, and supporting community people. In the context of Nepal, healthy dietary guidelines have been formulated, but still inadequacy of dietary guidelines for specific diseases condition as diabetes, cancer, hypertension and so on. Nevertheless, the government has formulated strategies making good and better health as ensuring adequate food access, promoting practices of good dietary habits in order to improve nutritional status, the action against infectious disease to improve child nutritional status and child mortality as well as school children, to control the incidence of lifestyle-related diseases as coronary artery disease, hypertension, tobacco and smoke-related diseases such as cancer, diabetes, dyslipidemia, etc. to reduce the critical risk of malnutrition and strengthening the system for analyzing, monitoring and evaluating the nutrition situation. There are lots of objectives. In spite of these, there is the inadequacy of dietary guidelines in the case of NCDs.

The fulfillment of objectives in NCDs that a multi-sector action plan has been designed to reduce morbidity, disability and premature mortality in Nepal. The targets taken to be fulfilled by 2020 are mortality reduced by $25 \%$ from cardiovascular disease, cancers, diabetes, or chronic respiratory diseases. It is targeted to reduce salt or sodium intake by $30 \%$ in the population. To reduce the prevalence of hypertension by $25 \%, 50 \%$ of the eligible people received drug therapy and counseling to prevent cardio diseases like heart attack and strokes. The fulfillment of the objective is concerned with the multisector organization as Ministry of Physical Infrastructure and Transport, Ministry of Education, Ministry of Home Affairs, Ministry of Urban Development, Ministry of Agricultural Development, Ministry of Federal Affair and Local Development, Ministry of Science, Technology, and Environment, Ministry of Health and Population, Civil Societies/NGOs and Private Enterprises.

WHO is leading and coordinating world health institutions for sustainable development agenda by 2030 as major challenges in recognizing NCDs. It has targeted to reduce one-third of premature mortality from NCDs through prevention and treatment which is mentioned in Sustainable Development Goal (SDG) 3.4. WHO developed a global action plan to prevent and control NCDs to support countries in their national efforts to reduce death by NCDs. The main target of this program is to approach (address) the prevention and management of NCDs by 2013-2020.

\section{Practices of Nutrition Counseling Service in the Hospital}

Nutrition counseling is the process of sharing nutrition and food knowledge with a person/patient whether in a disease condition or being healthy. So, nowadays, most of the hospital and health care centers had developed a concept of Wellness Clinic. The NDHS research, study knowledge among respondents was evaluated based on the pretest questionnaire. The result shows the literacy level of the low socioeconomic female has poor knowledge about disease condition. Therefore, to have a good recovery on NCDs hospital management should have the provision nutrition class so as to cover disease faster (Basu, 2017). Nevertheless, it is an individual's responsibility to take care of the diet being sick. Olukokun (2019) studied 'Pre and post-intervention of food in blood 
pressure patients' and found a positive effect in controlling blood pressure through food. This is a cohort study in the clinic among overweight and obese adult Nigerians showing the effect of nutrition counseling for hypertension. The number of NCDs is gradually being increased in Nepal. This study focused on the health strategy of the government and private sectors on strengthening preventive and curative health screening facilities on NCDs disease management (Shrestha, 2018).

\section{CONCLUSION AND IMPLICATION}

The lifestyle in Nepal has drastically been changed due to globalization and urbanization for decades. Its impacts are directly seen in food habits. Food diversity, sedentary lifestyle, stressful life and poor nutrition knowledge are the factors that contribute to NCDs. As WHO, the death in Nepal from NCD disease is 65\% out of 121,000 and the risk of premature death is $22 \%$ which is higher. Education, food availability and nutrition-awareness are the major impacting factors behind it. The ineffectiveness of unhealthy diet reduction measures and the use of alcohol reduction measures have less been taken as responses. So, the scenario of NCDs is being increased.

It is expected that the user of smoking will be reduced by 2025 in the same way obesity will be increased and the increased chances of hypertension. It shows that there are various preventive factors to be considered to reduce NCDs. Different stakeholders are the component to conduct campaigns on a healthy lifestyle as avoiding alcohol, discouraging advertisements to make tobacco-free environments as well as unhealthy food advertisement. The use of junk and calorie-dense food will result in overweight, obesity and NCDs, too. In the study carried out by Thakur (2016), there is an inadequate connection between adolescent health and national policies combating NCDs. However, nutrition counseling is one of the parts of the multidisciplinary approaches to controlling the diseases and it is a preventive measure as well. Therefore, there is a major role of Clinical Dietician in reducing NCDs diseases impacted by food habits.

Likewise, in the context of Nepal, the protocols prepared by the Ministry of Health and Population are inadequate in implementation. As per the protocol, there should be one Dietician at every hospital covered 50-75 beds. No one indeed stays in hunger without diet consultation, but nutrition counseling provides in-depth information on food, its effect on health and its impact on recovery. However, it has to be done because food contains lots of nutrition and should know the medicinal value of particular food regarding NCDs. For example, the diabetic patient is not allowed to have simple sugar, but on the hypoglycemic condition, patients are taught to have the same simple sugar. No doubt everyone is having/ taking meals as per individual's choice, but the role of a dietician is to recommend the whole day food amount as per the individual body condition will be fruitful. It helps with food mechanism and its positive impact on health. So, nutrition has a dual role in preventive and curative NCDs.

Therefore, the study suggests the protocol and guidelines prepared by the National Government and should strictly be followed, providing dietician roles so that nutritional healthy food habits can be created in order to prevent NCDs and nutrition policies. Every year Tribhuvan University is producing dieticians, but the need for proper mobilization is essential in order to make country people aware and healthy.

\section{REFERENCES}

American Heart Association. (2020). Body mass index in adults. What is the body mass index? https://www.heart.org/en/healthy-living/healthy-eating/losing-weight/ bmi-in-adults

Prithvi Academic Journal, Volume 4, May 2021 [pp. 121-132] $130 \mid \mathrm{P}$ a g e 
Arena, R., \& Guazzi, M. (2015). Healthy lifestyle interventions to combat NCDs disease-a novel nonhierarchical connectivity model for key stakeholders: A policy statement from the American Heart Association, European Society of Cardiology, European Association for Cardiovascular Prevention and Rehabilitation, and American College of Preventive Medicine. European Heart Journal, 36(31), 2097-2109.

Basu, S., Khobragade, M., Raut, K. K., \& Garq, S. (2017). Knowledge of diabetes among diabetic patients in government hospitals of Delhi. International Journal of NCDs Diabetes, 2(1), 8-10. https:// www.ijncd.org

Cao, Y., Willett, W. C., Rimm, E. B., Stampfer, M. J., \& Giovannucci, E. L. (2015). Light to

moderate intake of alcohol, drinking patterns, and risk of cancer: Results from two prospective US cohort studies. BMJ (Clinical research ed.), 351, 4238. https://doi.org/10.1136/bmj.h4238

CDC. (2017). Alcohol and public health. Centers for Diseases Control and Prevention. https://www.cdc.gov/alcohol/

CBS. (2011). Nepal living standards survey. Central Bureau of Statistics.

CDC. (2018). Smoking and tobacco use. Health effects of secondhand smoke. Centers for Disease Control and Prevention. https://www.cdc.gov/tobacco/ data_statistics/ fact_sheet

Chaudhary, D. (2018). Agricultural policies and rural development in Nepal: An overview. Research Nepal Journal of Development Studies, 1(2), 34-46. https://doi.org/10.3126/rnjds.v1i2.22425

Chaudhary D., et. al. (2021). Socioeconomic impacts and opportunities of COVID-19 for Nepal. In Khosla P.K., Mittal M., Sharma D., Goyal L.M. (eds). Predictive and Preventive Measures for Covid-19 Pandemic: Algorithms for Intelligent Systems. Springer. https://doi.org/10.1007/978-981-33-4236-1_9

Chauhan, S. A., \& Mukharjee, K. (2016). Economic burden of coronary heart disease in North India. International Journal of NCDs Diabetes, 1(1). 18-25. https:// www.ijncd.org

Heart Foundation. Is my blood pressure normal? https://www.heartfoundation.org.au/ your-heart/know-your-risks/blood-pressure/is-my-blood-pressure-normal

Liu, Y., Nguyen, N., \& Colditz, G. A. (2015). Links between alcohol consumption and breast

cancer: A look at the evidence. Women's health (London, England), 11(1), 6577. https://doi.org/10.2217/whe.14.62

Maharjan, K. (2019). Lifestyle factors and obesity in women. Hamro Sansar. A Journal of Women's Studies, 9. 28-30.

Maharjan, K., \& Chaudhary, D. (2021). Scenario and policy of decent nutrition and food

security in the Post-Covid-19 in Nepal. Journal La Sociale, 2(1), 10-17. https://doi.org/10.37899/journal-la-sociale.v2i1.251

Ministry of Health and Population Nepal (MoHP). (2018). Health video. Merobarsa: 5 commitments. https://www.mohp.gov.np/en/

Naya, F., Shatila, H., Meho,L., Alameddine, M., Haber, S., Nasreddine, L., Sibai, M. A., \& Hwalla, N. (2017). Gaps and opportunity for nutrition research in relation to NCDs diseases in Arab countries: Call for an informed research agenda. Elsevier, 47, 1-12. https://www.sciencedirect.com

Nepal nutrition and food security portal. Food temple poster_Nepal. http://nnfsp.gov.np/PortalCentent.aspx?Doctype=Resources\&ID=112 
Olukokun, A. T., Ayodapo, O. A., \& Monsudi, F. K. (2019). Effect of nutritional counseling on body mass index of adult hypertensive attending general outpatient clinic, federal medical center, Ido-Ekiti South-Western Nigeria. International Journal of NCDs Disease, 4(1). 15-20. https://www.ijncd.org

Sharma, A. (2017). Global research priorities for NCDs disease prevention, management, and control. International Journal of NCDs Diabetes, 2(4), 8107-112. https:// www.ijncd.org

Shrestha, B., Nepal, B., Mahat, R., \& Adhikari, A. (2018). Non-communicable disease pattern in adults seeking preventive general health checkup. Journal of Nepal Health Research Council (Grande Medical Journal), 1(1), https//www.nepjol.info

Stuetz, W. et al. (2019). Consumption of dark green vegetables predicts Vitamin A and Iron intake and status among female small scale farmers in Tanzania. https://www.ncbi.nlm.nih.gov

Thakur, S, J., Raina, N., Karna, P., Singh, P., Jeet, G., \& Jaswal, N. (2016). Overview of national strategy on NCDs diseases and adolescent health in South-East Asia Region countries. International Journal of NCDs Diabetes, 1(2), 76-86. www.ijncd.org

Tobacco control laws. Legislation of country Nepal. https://www.to baccocontrollaws.org/legislation/country/nepal/summary

Tokunaga, M. (2012). Diet, nutrients and NCDs diseases. The Open Nutraceutical Journal, 5, 146-159. https://benethamopen.com

WHO. (2002). Globalization, diets and non-communicable diseases. https://apps.who.int

WHO. (2018a). NCDs diseases (NCD) country profiles. Geneva: World Health Organization.

WHO. (2018b). Obesity and overweight. Facts about overweight. https://www.who.int/ news-room/fact-sheets/details/obesity-and-0verweight 